\title{
Evolução do Programa de Triagem Neonatal no Estado do Tocantins
}

\author{
Evolution of the Neonatal Screening Program in the State of Tocantins
}

Lucas Corrêa Mendes' ${ }^{1}$, Taides Tavares dos

Santos' ${ }^{\prime}$, Fabiana de Andrade Bringel'

${ }^{1}$ Faculdade de Ciências Humanas, Econômicas e da Saúde de Araguaína, Instituto Tocantinense Presidente Antônio Carlos (FAHESA) ITPAC), Araguaína, TO, Brasil

Correspondência para: Fabiana de Andrade Bringel Faculdade de Ciências Humanas, Econômicas e da Saúde de Araguaína Av. Filadélfia, 568, Setor Oeste 77816-540 - Araguaína, TO, Brasi cdfabiana@yahoo.com.br

Recebido em 17/Maio/2012 Aceito em 1/Out/2012

\section{RESUMO}

Objetivo: Avaliar o Programa de Triagem Neonatal do Estado do Tocantins de 1995 a 2011. Materiais e métodos: A coleta de dados foi realizada por meio de entrevista com os responsáveis pelo serviço, por análise de prontuários de pacientes com diagnóstico de fenilcetonúria (PKU) ou hipotireoidismo congênito $(\mathrm{HC})$ atendidos pelo programa e por entrevista com pais e/ou responsáveis por pacientes em acompanhamento. Resultados: A cobertura de triagem neonatal aumentou de $32,3 \%$ para $76,6 \%$ depois da implantação do Programa Nacional de Triagem Neonatal (PNTN). A prevalência de PKU e de HC no período analisado foi de 1:28.309 e de 1:4.632 nascidos vivos, respectivamente. A idade média das crianças na coleta da primeira amostra de sangue (PKU: 9,6 \pm 6,3 dias; HC: 13,3 \pm 10,3 dias) e no início do tratamento (PKU: 57,0 \pm 17,6 dias; HC: $95,6 \pm 57,6$ dias) foi superior às preconizadas pelo Ministério da Saúde. A avaliação dos pais sobre a qualidade do acompanhamento realizado foi classificada como satisfatória por $100 \%$ dos entrevistados. Conclusão: Embora tenha havido grande evolução do programa de triagem neonatal deste Estado, há necessidade de maior incentivo governamental para que o programa seja otimizado e possa avançar para as fases seguintes do PNTN. Arq Bras Endocrinol Metab. 2013;57(2):112-9

Descritores

Avaliação de serviços de saúde; fenilcetonúria; hipotireoidismo congênito

\section{ABSTRACT}

Objective: To evaluate the Neonatal Screening Program in the State of Tocantins from 1995 to 2011. Materials and methods: Data collection was conducted by means of interviews with those responsible for the service, by the analysis of medical records of patients diagnosed with phenylketonuria (PKU) and congenital hypothyroidism $(\mathrm{CH})$ that were enrolled in the program, and by interviews with parents and/or guardians of the patients monitored. Results: Program coverage increased from $32.3 \%$ to $76.6 \%$ after the implementation of the National Newborn Screening Program (PNTN). The prevalence of PKU and CH was 1:28,309 and 1:4,632 live births, respectively. The mean ages at the collection of the first blood sample (PKU: $9.6 \pm 6.3$ days; $\mathrm{CH}$ : $13.3 \pm 10.3$ days) and at the beginning of the treatment (PKU: $57.0 \pm 17.6$ days; $\mathrm{CH}: 95,6 \pm 57.6$ days) were greater than recommended by the Ministry of Health. The quality of monitoring was considered satisfactory by $100 \%$ of the parents. Conclusion: Although there have been great developments in neonatal screening program in this state, there is need for greater government incentives to optimize the program and to make the PNTN advance to its next phases. Arq Bras Endocrinol Metab. 2013;57(2):112-9

\section{Keywords}

Evaluation of health services; phenylketonuria; congenital hypothyroidism 


\section{INTRODUÇÃO}

$\mathrm{T}$ riagem significa seleção, separação de um grupo ou mesmo escolha entre inúmeros elementos e define, em saúde pública, a ação primária dos programas de triagem, ou seja, a detecção, por meio de testes aplicados em uma população, de um grupo de indivíduos com probabilidade elevada de apresentarem determinadas patologias. Quando essa metodologia de triagem é realizada especificamente na população com idade entre 0 e 30 dias de vida, aplica-se a definição triagem neonatal ( 1 ).

Entre os benefícios da triagem neonatal está a detecção de doenças graves e tratáveis antes do aparecimento dos sintomas, prevenindo problemas como retardo mental ou mesmo o óbito. Além disso, a identificação dos portadores de algumas doenças possibilita o aconselhamento genético e a reprodução consciente (2).

Conhecer a realidade e a prevalência das doenças que acometem a população neonatal favorece a elaboração e o aprimoramento das políticas públicas voltadas para a saúde da criança, minimiza gastos desnecessários em serviços de alta complexidade e contribui para a redução da taxa de mortalidade infantil (3).

Os Programas de Triagem Neonatal (PTN) são reconhecidos como eficazes e eficientes por prevenirem sequelas indeléveis quando as doenças são diagnosticadas e tratadas tempestiva e corretamente (1-3).

As doenças mais comumente diagnosticadas pelos PTN apresentam uma magnitude relativamente pequena, porém com potencial mórbido, incapacitante e causador de sequelas de tal ordem que tornam sua severidade e relevância social e econômica indiscutíveis (4).

A introdução dos PTN no Brasil, assim como em outros países em desenvolvimento, ocorreu de forma desorganizada, sem estrutura de controle da qualidade e precedeu a discussão ética em torno do assunto (5). Em 2001, foi criado pelo Ministério da Saúde o Programa Nacional de Triagem Neonatal (PNTN), executado pelos Serviços de Referência em Triagem Neonatal, que são instâncias ordenadoras e orientadoras de toda a rede estadual de triagem neonatal. O PNTN tem o objetivo de detectar, confirmar o diagnóstico, tratar e acompanhar os casos de até quatro doenças, conforme fases de implantação estabelecidas [Fase I: fenilcetonúria (PKU) e hipotireoidismo congênito (HC); Fase II: PKU, HC e hemoglobinopatias; Fase III: PKU, HC, hemoglobinopatias e fibrose cística] (1,4,6-8).
De acordo com o Ministério da Saúde, todos os estados brasileiros devem contar com pelo menos um Serviço de Referência em Triagem Neonatal (SRTN) e diversos postos de coleta distribuídos pelos municípios do Estado. As amostras sanguíneas devem ser obtidas corretamente e em tempo adequado com encaminhamento rápido ao laboratório, que deverá realizar os exames com rigoroso controle de qualidade (1). Quando um exame apresenta-se supostamente positivo, uma nova amostra de sangue deve ser solicitada e dirigida ao SRTN para confirmação diagnóstica. Se este não contar com exames de biologia molecular, deverá ainda enviar uma amostra de sangue para um laboratório que realize esses exames e que esteja credenciado em fase mais avançada do programa, conforme as normativas do Ministério da Saúde (1). Após a confirmação, realiza-se a segunda etapa da triagem neonatal, que, no Brasil, se denomina de busca ativa, que é o acompanhamento do resultado e localização do recém-nascido e sua família, já que o tempo do início do tratamento é crucial para que a mortalidade, a morbidade e as sequelas sejam prevenidas (2).

No Estado do Tocantins, a triagem neonatal é realizada desde 1995 por intermédio de iniciativa da Associação de Pais e Amigos dos Excepcionais (APAE) de Araguaína, que implantou um laboratório especializado para a realização dos exames em sua própria sede. Desde 2001, com a criação do PNTN, a APAE passou a sediar o SRTN do Tocantins, que, por estar na fase I do PNTN, realiza a triagem apenas de PKU e HC. PKU é um distúrbio autossômico recessivo do catabolismo da fenilalanina resultante de mutações no gene codificador da fenilalanina hidroxilase, enzima que converte fenilalanina em tirosina (9). O desenvolvimento do sistema nervoso central (SNC) é prejudicado pela hiperfenilalaninemia, acarretando manifestações clínicas que aparecem na segunda metade do primeiro ano de vida, tais como retardo do desenvolvimento neuropsicomotor, déficit de crescimento, irritabilidade, convulsões e eczema cutâneo, que são diretamente proporcionais aos níveis plasmáticos de fenilalanina (9-11). Hipotireoidismo é uma deficiência na produção ou na atuação dos hormônios tireoidianos (triiodotironina e tiroxina), sendo uma das doenças hormonais mais frequentes na pediatria (12). O HC deve-se à incapacidade da glândula tireoide do recém-nascido de produzir quantidades adequadas de hormônios tireoidianos, o que resulta numa redução generalizada dos processos metabólicos (1). 
Trabalhos já foram desenvolvidos em vários estados brasileiros (Bahia, Mato Grosso, Pará, Paraíba, Paraná, Santa Catarina, São Paulo, Sergipe) com o intuito de descrever e avaliar serviços de referência em triagem neonatal do Brasil e/ou traçar perfil epidemiológico de doenças triadas por estes $(3,7,13-20)$. Entretanto, trabalhos como esses nunca foram realizados no Programa de Triagem Neonatal do Estado do Tocantins.

É fundamental que sejam realizadas avaliações periódicas da qualidade dos programas de triagem neonatal para que eles consigam atingir plenamente seus objetivos, reportando-se os resultados dessas avaliações às autoridades públicas responsáveis, de modo que eventuais modificações possam ser implementadas para aprimorá-lo (19). Dessa forma, o presente trabalho objetivou avaliar o programa de triagem neonatal do Estado do Tocantins de 1995 a 2011.

\section{MATERIAIS E MÉTODOS}

Foi realizado estudo descritivo transversal com base no banco de dados do Laboratório da APAE de Araguaína, sede do SRTN do Estado do Tocantins, e em entrevista com os responsáveis pelos pacientes em acompanhamento.

Este trabalho respeitou as Resoluções n ${ }^{\circ}$ 196/96 e 251/97 do Conselho Nacional de Saúde. A autorização da pesquisa foi concedida pelo gestor da APAE de Araguaína e o trabalho foi aprovado pelo Comitê de Ética em Pesquisa da Fundação de Medicina Tropical do Tocantins (processo $n^{\circ} 085 / 2010$ ). Os responsáveis pelos pacientes em acompanhamento concordaram em participar mediante a assinatura do Termo de Consentimento Livre e Esclarecido.

A análise do banco de dados e entrevista com os responsáveis pelas crianças assistidas pelo serviço foi realizada no período de janeiro a dezembro de 2011.

Como critério de inclusão utilizaram-se todos os recém-nascidos vivos no período de março de 1995 a dezembro de 2011 e que realizaram o teste de triagem na rede pública do Estado do Tocantins, cujo resultado das amostras de sangue, coletadas em papel filtro, tenha dado positivo para PKU ou HC. As dosagens de TSH foram feitas pelo método imunofluorimétrico utilizando o kit da DELFIA ${ }^{\circledR}$ (Perkin-Elmer, Wallac Oy, Turku, Finlândia), sendo considerados como normais os níveis abaixo de $20 \mathrm{mUI} / \mathrm{L}$ de sangue e as de fenilalanina, pelo método fluorimétrico, sendo o valor de referência da triagem para a população normal menor ou igual a
$4 \mathrm{mg} \%$; ambas as dosagens foram feitas utilizando-se o equipamento Victor ${ }^{2}$ D (Perkin-Elmer, Wallac Oy, Turku, Finlândia).

Foram excluídas da pesquisa duas crianças portadoras de PKU e três crianças portadoras de $\mathrm{HC}$ porque o diagnóstico foi feito antes da implantação do programa de triagem neonatal no Tocantins. Quatro pacientes com HC foram excluídos porque o diagnóstico foi feito fora do SRTN do Tocantins, além de três crianças que foram diagnosticadas com $\mathrm{HC}$ transitório. Os responsáveis por um paciente com PKU e oito pacientes com HC não foram entrevistados devido à localização desconhecida e por não frequentarem o serviço de acompanhamento.

As variáveis analisadas foram: avaliação da estrutura organizacional e qualidade do serviço, número de crianças nascidas vivas no estado (dado fornecido pelo Sistema de Informações sobre Nascidos Vivos [Sinasc]), taxa de cobertura da triagem neonatal, gênero das crianças com diagnóstico positivo para PKU e HC, idade da criança na primeira coleta, tempo decorrido entre a coleta e a chegada da amostra ao laboratório e da emissão do resultado confirmatório, e idade da criança no início do tratamento.

Para a avaliação da estrutura organizacional do serviço, foi realizada entrevista com preenchimento de um questionário com os responsáveis pelo programa. Nesse questionário foi possível identificar em que fase do PNTN o serviço se encontrava, como as amostras eram encaminhadas para o laboratório, como os resultados eram encaminhados aos municípios de origem das amostras, qual a cobertura geográfica do programa e a quantidade de postos de coleta no Estado.

Quanto à qualidade prestada pelo serviço, foi realizada uma entrevista com os responsáveis pelas crianças acompanhadas, no intuito de qualificar o acompanhamento durante o tratamento e de obter informações quanto à existência de repasse, por parte do SRTN, de esclarecimentos sobre a doença diagnosticada e suas possíveis complicações.

As demais variáveis foram obtidas por meio da análise dos prontuários de pacientes com diagnóstico de PKU ou de HC, atendidos pelo programa de triagem neonatal do Estado no período anteriormente citado.

Os dados foram tabulados em planilha eletrônica onde foram obtidas as variáveis estatísticas (média, desvio-padrão, mediana, valor máximo e valor mínimo) em programa Excel, versão 2010 (Microsoft $\left.{ }^{\circledR}\right)$. 


\section{RESULTADOS}

Por meio da avaliação da estrutura organizacional do serviço prestado no Tocantins, foram identificados 238 postos de coleta credenciados, distribuídos em 125 municípios. O Estado possui 139 municípios, correspondendo a $89,9 \%$ de cobertura geográfica estadual. O programa dispõe de um laboratório especializado localizado em Araguaína, responsável pelos testes de triagem, analisando amostras de sangue advindas de todos os postos de coleta credenciados.

As amostras sanguíneas são encaminhadas pelas unidades de saúde ao laboratório por malotes ou por motoristas de ambulância que conduzem pacientes até a cidade sede do serviço; os resultados são enviados pelas mesmas vias. Quando um exame apresenta-se supostamente positivo para PKU ou HC, duas novas amostras de sangue são solicitadas, uma para confirmação diagnóstica pelo próprio PTN do Tocantins e outra para confirmação pelo laboratório de Anápolis, no Estado de Goiás, uma vez que este está na fase II do programa.

O tratamento e acompanhamento nesse serviço dos casos de PKU e HC são realizados por equipe multidisciplinar, composta por assistente social, biomédico, enfermeiro, farmacêutico bioquímico, médico endocrinologista, médico pediatra e nutricionista. A APAE de Araguaína dispõe ainda de outros profissionais que não são vinculados ao SRTN, como cirurgião-dentista, farmacêutico, fisioterapeuta, fonoaudiólogo e terapeuta ocupacional, que prestam assistência de apoio aos pacientes do SRTN de acordo com a necessidade.

No que se refere à cobertura de triagem neonatal, na figura 1 é demonstrada a evolução desta no período de 1995 a 2011. A partir dos dados descritos na figura 1 , calculou-se a cobertura desse programa antes (1995-2000) e depois (2001-2011) da implantação do PNTN, que foi de $32,3 \%$ e $76,6 \%$, respectivamente.

Do total de nove pacientes com PKU, três são do sexo masculino $(33,33 \%)$ e seis, do sexo feminino $(66,66 \%)$. Em relação ao HC, dos 55 casos detectados, 21 são do sexo masculino $(38,18 \%)$ e $34(61,81 \%)$ são do sexo feminino, como se pode verificar na tabela 1 , que considera apenas pacientes nascidos entre $1995 \mathrm{e}$ 2011.

Considerando o total de nascidos vivos triados entre 1995 e 2011 (254.782) e o número de diagnósticos nesse mesmo período (nove pacientes com PKU e 55 com HC), determinou-se a prevalência de 1:28.309 para PKU e de 1:4.632 para HC no programa do Estado do Tocantins.

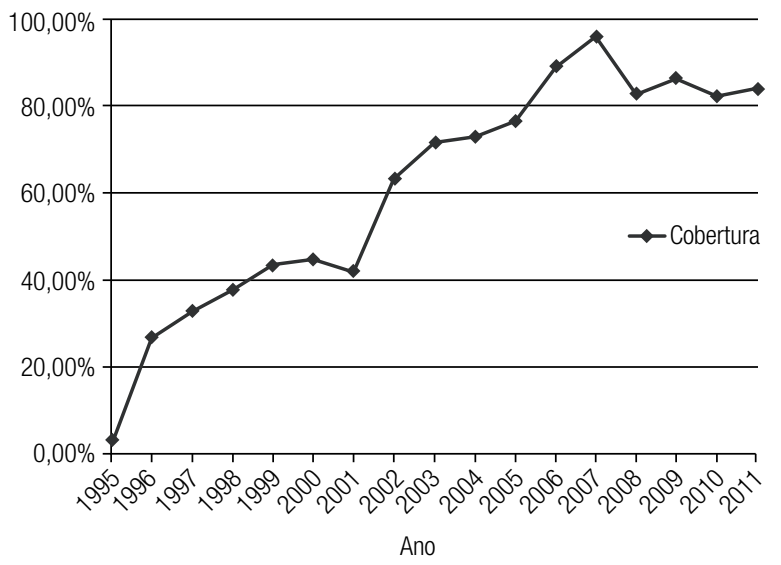

Figura 1. Cobertura do Programa de Triagem Neonatal do Tocantins no período de 1995 a 2011. No eixo horizontal, temos o período no qual a cobertura foi verificada e, no eixo vertical, os valores de cobertura em porcentagem. Fonte: APAE, 2011.

Tabela 1. Casos de PKU e HC detectados pelo Programa de Triagem Neonatal do Tocantins entre 1995 e 2011

\begin{tabular}{lccccc}
\hline \multirow{2}{*}{ Ano } & \multicolumn{2}{c}{$\begin{array}{c}\text { Número de casos de PKU } \\
\text { (Segundo o gênero) }\end{array}$} & & \multicolumn{2}{c}{$\begin{array}{c}\text { Número de casos de HC } \\
\text { (Segundo o gênero) }\end{array}$} \\
\cline { 2 - 3 } \cline { 5 - 6 } & Masculino & Feminino & & Masculino & Feminino \\
\hline 1996 & 0 & 1 & & 0 & 0 \\
1998 & 0 & 1 & & 0 & 0 \\
1999 & 0 & 0 & & 0 & 1 \\
2000 & 2 & 0 & & 0 & 0 \\
2002 & 1 & 0 & & 0 & 1 \\
2003 & 0 & 0 & & 2 & 4 \\
2004 & 0 & 1 & & 2 & 3 \\
2005 & 0 & 0 & & 5 & 2 \\
2006 & 0 & 0 & & 2 & 3 \\
2007 & 0 & 0 & & 2 & 3 \\
2008 & 0 & 1 & & 1 & 3 \\
2009 & 0 & 2 & & 0 & 3 \\
2010 & 0 & 0 & & 4 & 2 \\
2011 & 0 & 0 & 2 & 5 \\
Indefinido* & 0 & 0 & & 1 & 4 \\
Total & 3 & 6 & 21 & 34 \\
\hline
\end{tabular}

* Pacientes cujo ano de diagnóstico é desconhecido. Fonte: APAE, 2011.

A partir da análise de dados disponíveis nos prontuários de pacientes com diagnóstico de PKU ou HC atendidos pelo programa, foi verificado que a média do tempo decorrido entre o nascimento da criança e a coleta da primeira amostra foi de 9,6 $\pm 6,3$ dias $(\mathrm{N}=7$; mediana $=6$; range $=3$-19) para os pacientes com PKU e de $13,3 \pm 10,3$ dias $(\mathrm{N}=46 ;$ mediana $=10 ;$ range $=$ 3-54) para os pacientes com $\mathrm{HC}$; a idade das crianças no início do tratamento foi de 57,0 $\pm 17,6$ dias 
$(\mathrm{N}=4 ;$ mediana $=59,0 ;$ range $=38-72)$ para as diagnosticadas com PKU e de 95,6 \pm 57,6 dias $(\mathrm{N}=43$; mediana $=80,0$; range $=38-304)$ para as diagnosticadas com HC.

Quanto à qualidade da assistência fornecida pelo SRTN do Estado, cem por cento dos responsáveis entrevistados $(\mathrm{N}=8$ para $\mathrm{PKU}$ e $\mathrm{N}=41$ para $\mathrm{HC})$ consideram que seus filhos ou dependentes dispõem de bom atendimento. Quando os entrevistados foram questionados sobre a existência de repasse, por parte do SRTN, de informações sobre a causa, o tratamento e possíveis complicações das patologias envolvidas nesse trabalho, todos afirmaram ter recebido os devidos esclarecimentos.

Solicitou-se aos entrevistados que apontassem falhas do serviço de referência ou apresentassem sugestões para melhorá-lo. Entre os responsáveis pelos pacientes com PKU, houve unanimidade em afirmar que as falhas inexistiam; como sugestão, foi apontada por um entrevistado a necessidade de se disponibilizar serviço de psicologia em sua cidade. Entre os de HC, um responsável apontou falha na coleta de sangue para teste de controle em seu município; outro apontou dificuldade na emissão de resultados dos exames de controle em tempo hábil para consulta. As seguintes sugestões foram apresentadas: descentralizar o local de consultas, viabilizar o deslocamento da cidade de origem até a sede do serviço e facilitar o fornecimento gratuito de exames de controle.

\section{DISCUSSÃO}

Dado o ineditismo desse tipo de estudo no Estado do Tocantins, a tabulação e análise das informações coletadas permitiram a construção de um banco de dados sistematizado referente ao programa, que favorecerá a gestão do serviço e poderá servir como instrumento de consulta para avaliações posteriores desse ou de outros programas de triagem neonatal brasileiros. Além disso, a realização desse trabalho tornou possível a divulgação dos resultados sobre a triagem neonatal realizada no Tocantins, enfocando sua importância no diagnóstico precoce de anomalias congênitas e na promoção de tratamento a tempo de se evitar complicações sérias como o retardo mental.

O SRTN do Tocantins, assim como o da Bahia, Espírito Santo, Goiás, Maranhão, Mato Grosso do Sul e São Paulo, tem sede na APAE. Nos outros estados brasileiros, os serviços de referência em triagem neonatal são sediados em núcleos de atendimento, hospitais, maternidades, fundações e outras entidades (2l).

No que se refere à rede de coleta da triagem neonatal do Tocantins, foi observada uma cobertura geográfica estadual de $71,6 \%$ em 2003, valor aquém ao encontrado, no mesmo ano, no Estado do Mato Grosso (90\%) (20) e no Estado da Bahia (94,5\%) (7).

Com a criação do PNTN em 2001, houve elevação da cobertura de triagem neonatal no Brasil para aproximadamente $80,0 \%$ em 2005 , porém, de forma bastante heterogênea entre os Estados $(4,20,22)$; por isso, para esse estudo, optou-se em dividir o período de análise em duas partes, de 1995 a 2000 (antes de ser SRTN) e de 2001 a 2011 (após virar SRTN), objetivando verificar sua evolução nesses dois períodos. Observou-se um aumento considerável da cobertura entre 1995, ano de criação do programa, e o ano seguinte, passando de $2,9 \%$ para 26,9\%; em 2001, quando foi implantado o PNTN, foi igual a $41,9 \%$ e $63,3 \% \log$ o em 2002 ; os anos com maior índice de cobertura foram $2006 \mathrm{e}$ 2007 , com $89,1 \%$ e $96,2 \%$, respectivamente; o ano de 2010 , com $82,2 \%$ de cobertura, apresentou o menor percentual de crianças triadas; no ano seguinte, a cobertura foi de $84,2 \%$.

A cobertura populacional total é muitas vezes dificultada por falta de implantação de postos de coleta. Dos 139 municípios do Tocantins, 14 não possuem postos de coleta. Também existem problemas socioeconômicos e culturais, falta de informações da importância do teste de triagem neonatal, além de vários fatores que dificultam o acesso aos serviços de saúde, principalmente dos municípios ao norte do Estado: a extensão territorial do estado de Tocantins, a localização geográfica de alguns municípios com grande parte da população na zona rural onde o acesso aos postos de coleta para a realização dos exames é mais difícil, além das péssimas condições nas estradas nos períodos de chuva. As oscilações na taxa de cobertura se devem, ainda, ao fato de que grande parte dos funcionários responsáveis pela coleta da amostra e realização dos exames não é efetiva e a perda frequente de indivíduos treinados demanda tempo para treinamento de novos profissionais.

Neste estudo, verificou-se uma cobertura de 32,3\% no primeiro período e de 76,6\% no segundo. Em estudos realizados no Estado de Sergipe no ano de 1995 (23) e em 2003 (16), foram apresentadas coberturas da triagem neonatal de $15,0 \%$ e $72,0 \%$, respectivamente, demonstrando uma considerável evolução após a implantação do PNTN, assim como ocorreu no Tocan- 
tins. Em Santa Catarina, foi demonstrada uma cobertura de $81,0 \%$ entre 1994 e 1998 , chegando a $91,4 \%$ no ano de 1997, mesmo antes da implantação do PNTN (13), sendo que no Estado do Mato Grosso ela foi de $65,3 \%$ e $67,6 \%$ em 2003 e 2004 , respectivamente, caracterizando-se um discreto incremento entre os anos analisados (20). No Paraná, entre 1996 e 2001, foi verificada uma cobertura de $92,4 \%$ (15).

O programa de triagem do Tocantins, assim como os outros programas citados, não atingiu a cobertura de 100\%, preconizada com a criação do PNTN em 2001. Porém, nota-se um incremento significativo após a implantação do SRTN, que está relacionado com uma melhor estruturação do serviço, favorecendo, dessa forma, uma triagem mais efetiva de PKU e HC no Estado.

A equipe multidisciplinar mínima necessária para o funcionamento de um SRTN é simples, tendo uma pequena variação de acordo com a patologia a ser acompanhada, sendo composta de médico pediatra, nutricionista, psicólogo e assistente social para o acompanhamento de pacientes fenilcetonúricos, e médico endocrinologista/endocrinologista pediátrico, psicólogo e assistente social para pacientes com HC (1). O programa de triagem neonatal do Estado do Tocantins cumpre essa prerrogativa.

Quanto à prevalência das doenças rastreadas no Tocantins, no período analisado, uma prevalência de 1:28.309 nascidos vivos foi detectada para PKU, sendo menor que a encontrada, em 2002, para o Brasil, de 1:24.780 nascidos vivos (24). A prevalência encontrada no Tocantins foi maior que a encontrada no Paraná (1:34.499), em estudo que avaliou o período de 1996 a 2001 (15) e maior que a do Mato Grosso em 2003, de 1:33.068 nascidos vivos (20).

A SBTN verificou a prevalência decrescente para a doença, 1:16.691 em 2001 e 1:24.310 nascidos vivos em 2002, em estudos realizados com 20 serviços de referência em triagem neonatal em 15 estados brasileiros no período de 2001 a 2002 (25); a prevalência de PKU nesse estudo para o ano de 2002 é maior do que a encontrada no programa do Tocantins.

A prevalência de HC encontrada no programa do Tocantins foi de 1:4.632 nascidos vivos; em estudo semelhante, em que foi avaliado o programa de triagem neonatal do Estado de Sergipe, de 1998 a 2003, foi calculada uma prevalência de 1:4.850 nascidos vivos (16), próxima à encontrada no programa do Tocantins. Entre 1994 e 1998, houve uma prevalência de 1:3.177 nascidos vivos para HC no programa de Santa Catarina
(13). No estudo realizado pela SBTN, anteriormente citado, foram encontradas prevalências para $\mathrm{HC}$ de l:3.694 nascidos vivos em 2001 e de 1:3.808 em 2002 (25), maiores que a encontrada nesta análise.

O principal objetivo da triagem neonatal é realizar o teste em tempo hábil, viabilizando o adequado início do tratamento e a prevenção de deficiência mental e outras sequelas (11). O atraso em qualquer etapa de triagem neonatal (coleta, chegada da amostra no laboratório, emissão dos resultados, entre outras) pode acarretar prejuízos permanentes para a vida da criança acometida pela doença e sua família (3). O Ministério da Saúde estabelece como meta a realização da coleta de sangue para a triagem entre o terceiro e o sétimo dia de vida (1). No Tocantins, foi verificado que os pacientes com PKU e HC tiveram amostras coletadas com idade média de 9,6 $\pm 6,3$ dias e $13,3 \pm 10,3$ dias, respectivamente. Em trabalhos que avaliaram todos os recém-nascidos triados no serviço público do município de Campina Grande, Paraíba, entre 2000 e 2001, verificou-se idade média do paciente no momento da coleta de $18,2 \pm 12,2$ dias (14), e no Estado de Sergipe, em 2003, a idade média das crianças na coleta foi de $12 \pm 11$ dias (16). Em todos os locais mencionados, as coletas foram realizadas em tempo superior ao preconizado.

No que se refere à idade de início do tratamento, verificou-se que a idade da criança no início do tratamento, no Estado do Tocantins, era em média de $57 \pm$ 17,6 dias $(\mathrm{N}=4$, mediana $=59$ dias, variando de 38 a 72 dias) para PKU. Para HC era de 95,6 $\pm 57,6$ dias (N $=43$, mediana $=80$ dias, variando de 38 a 304 dias . Em Santa Catarina, em uma avaliação que considerou dados de 1994 a 1998 do programa de triagem neonatal para $\mathrm{HC}$, a idade das crianças na primeira consulta e início do tratamento foi de 40,2 $\pm 15,9$ dias $(\mathrm{N}=$ 123, mediana $=38,0$ dias, $13-101$ dias) (13). Portanto, nenhum dos dois estados cumpre o recomendado pelo Ministério da Saúde, de que o tratamento seja instituído no primeiro mês de vida do recém-nascido (1). No estudo que considerou dados de 1994 a 2005 do programa de triagem neonatal do Hospital das Clínicas da Faculdade de Medicina de Ribeirão Preto, foi verificado que a idade das crianças com HC foi de 25,9 $\pm 14,2$ dias $(\mathrm{N}=60$, mediana $=22,5$ dias, $10-86$ dias $)$ e com PKU foi de $25,7 \pm 17,5$ dias $(\mathrm{N}=10$, mediana $=19,5$ dias, 9-60 dias) (19), atingindo o tempo preconizado já mencionado. Segundo dados obtidos da Sociedade Brasileira de Triagem Neonatal, que avaliou a realidade 
de 18 unidades da federação, exceto Tocantins, a idade média da criança no início do tratamento era de 34,0 \pm 15 dias para $\mathrm{PKU}$ e de $34,0 \pm 17$ dias para $\mathrm{HC}$, no período de 2001-2002 (25), lembrando que se trata da média nacional e que cada unidade da federação possui características regionais distintas, englobando fatores socioeconômicos e culturais, já citados, que influenciam muito os resultados obtidos no programa de triagem.

Portanto, verificamos que tanto a idade da criança na coleta da amostra quanto a idade desta no início do tratamento estão acima da idade preconizada. Segundo os responsáveis pelo PTN do Estado do Tocantins, uma das razões seria a constante troca de funcionários nos postos de coleta, visto que a grande maioria não é efetiva, o que implica dificuldade de treinamento e coleta de amostras inadequadas. A necessidade de se repetir a coleta gera resistência dos familiares e aumenta o tempo para se realizar o diagnóstico e se iniciar o tratamento.

A ultrapassagem do tempo recomendado no programa do Tocantins pode ainda ser justificada pela retenção de amostras no posto de coleta para posterior envio e a necessidade de se percorrer grandes distâncias até o laboratório. Com o atraso da chegada ao laboratório, adia-se também a emissão do resultado, aumentando o tempo decorrido até o início do tratamento.

A partir do exposto, percebe-se que os aspectos relacionados à condução dos testes de triagem podem e devem ser melhorados, a fim de se diminuir a idade de coleta, do tempo decorrido entre a coleta e a chegada da amostra ao laboratório e da emissão do resultado confirmatório, diminuindo, dessa forma, a idade de início do tratamento. Verifica-se, então, a necessidade de se criarem estratégias visando reduzir esse tempo no programa de triagem neonatal desse Estado. O aprimoramento da rede de coleta e maior abrangência desta, divulgação da triagem neonatal perante a população e os profissionais de saúde na intenção de conscientizá-los da importância deste, visando atingir a cobertura universal da população neonatal, são algumas sugestões de medidas a serem adotadas.

A assistência dedicada aos pacientes com PKU ou HC cadastrados no SRTN, por ter sido classificada como boa ou excelente por todos os responsáveis entrevistados, foi considerada satisfatória, ainda que alguns desses, isoladamente, tenham apontado falhas relacionadas à coleta e à emissão de resultados em tempo hábil para consulta. Visto que, após a confirmação do diagnóstico das duas patologias, existe o devido esclarecimento aos responsáveis pelos pacientes sobre es- tas quanto à forma de tratamento e suas complicações, projetam-se uma melhor aceitação e eficácia no tratamento, diminuindo ou anulando as possíveis sequelas decorrentes das patologias.

Conclui-se que o Programa de Triagem Neonatal do Estado do Tocantins atende aos requisitos mínimos recomendados pelo Ministério da Saúde e nossos dados indicam uma evolução significativa da taxa de cobertura, revelando prevalência de PKU e HC no Estado do Tocantins menor do que a nacional. No entanto, a coleta da primeira amostra de sangue e o início do tratamento ocorreram em tempo superior ao preconizado pelo Ministério da Saúde.

Apesar da percepção positiva dos pais sobre a qualidade do acompanhamento realizado por esse programa de triagem neonatal e a grande evolução deste desde sua implantação, há necessidade de maior incentivo governamental para que tal programa seja otimizado e possa avançar para as fases seguintes do PNTN.

Agradecimentos: à Faculdade de Ciências Humanas, Econômicas e da Saúde de Araguaína, Instituto Tocantinense Presidente Antônio Carlos (FAHESA/ITPAC), pelo apoio financeiro concedido por meio do Programa Institucional de Bolsas de Iniciação Científica, e ao Serviço de Referência em Triagem Neonatal do Estado do Tocantins, por permitir a realização deste trabalho em suas dependências.

Declaração: os autores declaram não haver conflitos de interesse científico neste estudo.

\section{REFERÊNCIAS}

1. Ministério da Saúde. Manual de normas técnicas e rotinas operacionais do Programa Nacional deTriagem Neonatal, Brasília (DF); 2004.

2. Leão LL, Aguiar MJ. Newborn screening: what pediatricians should know. J Pediatr (Rio J). 2008;84(4 Suppl):S80-90.

3. Luz GS, Carvalho MDB, Pelloso SM, Higarashi IH. Prevalência das doenças diagnosticadas pelo Programa de Triagem Neonatal em Maringá, Paraná, Brasil: 2001-2006. Rev Gaúcha Enferm. 2008;29(3):446-53.

4. Botler J, Camacho LAB, Cruz MM, George P.Triagem neonatal - o desafio de uma cobertura universal e efetiva. Ciênc Saúde Coletiva. 2010;15(2):493-508.

5. Souza CFM, Schwartz IV, Giugliani R. Triagem neonatal de distúrbios metabólicos. Ciênc Saúde Coletiva. 2002;7(1):129-37.

6. Horovitz DDG, Llerena Júnior JC, Mattos RA. Atenção aos defeitos congênitos no Brasil: panorama atual. Cad Saúde Pública. 2005;21(4):1055-64.

7. Almeida AM, Godinho TM, Teles MS, Rehem APP, Jalil HM, FukudaTG, et al. Avaliação do Programa deTriagem Neonatal na Bahia no ano de 2003. Rev Bras Saúde Matern Infant. 2006;6(1):85-91.

8. Ramalho ARO, Ramalho RJR, Oliveira CRP, Santos EG, Oliveira $\mathrm{MCP}$, Aguiar-Oliveira MH. Programa de triagem neonatal para hipotireoidismo congênito no Nordeste do Brasil: critérios diagnósticos e resultados. Arq Bras Endocrinol Metab. 2008;52(4):617-27. 
9. Nussbaum RL, Mclnnes RR, Willard HF. Thompson \& Thompson Genética Médica. 7. ed. Elsevier: Rio de Janeiro (RJ); 2008. p. 525.

10. Pires RF. Aminoacidopatias. In: Carakushansky G. Doenças genéticas em pediatria. Guanabara Koogan S. A.: Rio de Janeiro (RJ); 2001. p. 159-61.

11. Amorim T, Gatto SPP, Boa-Sorte N, Leite MEQ, Fontes MIMM, Barretto $\mathrm{J}$, et al. Aspectos clínicos da fenilcetonúria em serviço de referência em triagem neonatal da Bahia. Rev Bras Saúde Matern Infant. 2005;5(4):457-62.

12. Setian N. Hypothyroidism in children: diagnosis and treatment. J Pediatr. 2007;83(5):209-16.

13. Nascimento ML, Pires MMS, Nassar SM, Ruhland L. Avaliação do programa de rastreamento neonatal para hipotireoidismo congênito da Secretaria de Estado da Saúde de Santa Catarina. Arq Bras Endocrinol Metab. 2003;47(1):75-81.

14. Ramos AJS, Rocha AM, Costa ADM, Benicio AVL, Ramos ALC, Silva $C R A$, et al. Avaliação do programa de rastreamento de doenças congênitas em Campina Grande - PB, Brasil. Arq Bras Endocrinol Metab. 2003;47(3):280-84.

15. Brandalize SRC, Czeresnia D. Avaliação do programa de prevenção e promoção da saúde de fenilcetonúricos. Rev Saúde Pública. 2004;38(2):300-6.

16. Ramalho RJR, Ramalho ARO, Oliveira CRP, Aguiar-Oliveira MH. Evolução do programa de triagem neonatal para o hipotireoidismo congênito e fenilcetonúria no Estado de Sergipe de 1995 a 2003. Arq Bras Endocrinol Metab. 2004;48(6):890-6.

17. Benevides AM, Lima CHV, Rocha CA, Corrêa ARR, El Husny AS, Fernandes-Caldato MC. Perfil epidemiológico de portado- res de hipotireoidismo congênito. Rev Paraense de Medicina. 2006;20(3):23-6.

18. Carvalho MDB, Pelloso SM, Higarashi IH, Luz GS. Cobertura do programa de triagem neonatal em Maringá (PR), 2001 a 2006. Acta Paul Enferm. 2008;21(1):89-93.

19. Magalhães PKR, Turcato MF, Angulo IL, Maciel LMZ. Programa de triagem neonatal do Hospital das Clínicas da Faculdade de Medicina de Ribeirão Preto, Universidade de São Paulo, Brasil. Cad Saúde Pública. 2009;25(2):445-54.

20. Stranieri I,Takano OA. Avaliação do serviço de referência em triagem neonatal para hipotireoidismo congênito e fenilcetonúria no Estado de Mato Grosso, Brasil. Arq Bras Endocrinol Metab. 2009;53(4):446-52.

21. Sociedade Brasileira de Triagem Neonatal. Triagem. Locais de Exame - Público. Disponível em: <http://www.sbtn.org.br/pg triag_oquee_locexame_publico.htm>Acesso em: 30 Out, 2011.

22. CarvalhoTM. Triagem neonatal no Brasil. Rev Med Minas Gerais. 2005;15(Suppl.1):20-2.

23. Ramalho RJR, Valido DP, Aguiar-Oliveira MH. Avaliação do programa de triagem para o hipotireoidismo congênito no Estado de Sergipe. Arq Bras Endocrinol Metab. 2000;44(2):157-61.

24. Carvalho TM, Santos HP, Santos IC, Vargas PR, Pedrosa J. Newborn screening: a national public health programme in Brazil. $J$ Inherit Metab Dis. 2007;30:615.

25. Carvalho TM, Pimentel H, Carvalho GPM. Levantamento epidemiológico brasileiro de triagem. Disponível em: <http://www. sbtn.org.br/images/dados/dados_2002_2003. swf > Acesso em: 18 Jul, 2012. 